\title{
TETRAETHYLENE GLYCOL TETHERED HOMONUCLEAR AND HETERONUCLEAR ISATIN DIMERS AND THEIR IN VITRO ANTI-MYCOBACTERIAL ACTIVITIES
}

\author{
Yin-Ling WANG, ${ }^{\text {,a }}$ Shi-Jia ZHAO, ${ }^{\mathrm{b}}$ Jia-Lun Deng, ${ }^{\mathrm{c}}$ Zao-Sheng LV ${ }^{\mathrm{b}}$ and Zhi XU,a \\ ${ }^{a}$ Huanghuai University, Industry Innovation \& Research and Development Institute of Zhumadian, \\ Kaiyuan Road, Zhumadian, 463000, Henan, P. R. China \\ ${ }^{b}$ Key Laboratory of Hubei Province for Coal Conversion and New Carbon Materials, Wuhan University of Science and Technology, \\ Heping Avenue Qingshan District, Wuhan, 430081, Hubei, P. R. China \\ ${ }^{\mathrm{c}}$ Haiso Technology Co., Ltd., Wuhan, 430074, P. R. China
}

Received May 31, 2018

A series of novel homonuclear and heteronuclear isatin dimers tethered via tetraethylene glycol were synthesized and assessed for their in vitro antimycobacterial activities against MTB H37Rv and MDR-TB as well as cytotoxicity in VERO cells. The preliminary results showed that all dimers had potential antimycobacterial activities and acceptable cytotoxicity. Amongst them, the heteronuclear isatin dimer 10f (MIC: 32 and $32 \mu \mathrm{g} / \mathrm{mL}$ ) was found to be most active against MTB H37Rv and MDR-TB strains, which was as potent as or better than the first-line anti-tubercular agents rifampicin (MIC: $32 \mu \mathrm{g} / \mathrm{mL}$ ) and isoniazid (MIC: $>128 \mu \mathrm{g} / \mathrm{mL}$ ) against MDR-TB, also demonstrated excellent cytotoxicity profile $\left(\mathrm{CC}_{50}: 250 \mu \mathrm{g} / \mathrm{mL}\right)$.

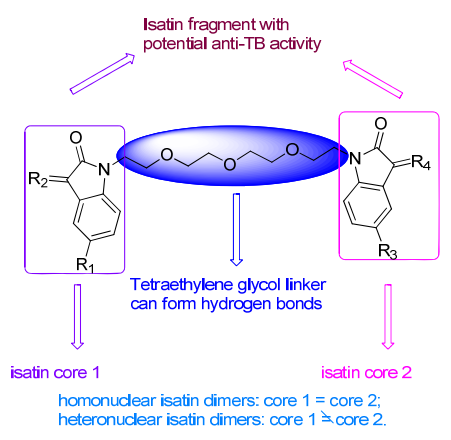

\section{INTRODUCTION}

Tuberculosis (TB) is a highly infectious deadliest disease caused predominately by Mycobacterium tuberculosis (MTB), and affects mainly the lungs (pulmonary TB) ${ }^{1,2}$ The World Health Organization (WHO) has estimated that roughly 2 billion people harbor latent MTB infection globally, and around 10.4 million people fell ill and 1.67 million deaths in $2016 .^{1}$

The first-line anti-TB agents such as isoniazid (INH), rifampicin (RIF), pyrazinamide (PZA) and ethambutol (EMB) play a pivotal role in the treatment of drug-susceptible MTB infected patients. $^{1}$ However, the reemergence of TB infection is complicated by the evolution of MTB new virulent forms including drug-resistant TB (DR-TB), multidrug-resistant TB (MDR-TB), extremely drug-resistant TB (XDR-TB) and totally drug resistant TB (TDR-TB). ${ }^{3-5}$ Only a handful of candidates have entered human trials after the discovery of RIF, and the number is obviously insufficient. ${ }^{4}$ All the above facts necessitated an urgent need to develop new anti-TB agents which are effective against both drug-susceptible and drug-resistant TB.

Isatins possess diverse biological and pharmacological properties such as antibacterial, ${ }^{6,7}$ anticancer, ${ }^{8}$ antimalarial ${ }^{9}$ and anti-TB activities, ${ }^{10-14}$ and isatin dimers also showed considerable antiTB activity. ${ }^{15-17}$ The structure-activity relationship (SAR) of isatin dimers revealed that the linker between the two isatins is crucial for their anti-TB

\footnotetext{
*Corresponding author: chemorgchem@126.com
} 
activity, ${ }^{18,19}$ so it's reasonable to optimize the linker. Tetraethylene glycol fragment has the potential to form hydrogen bonds that facilitate binding with active site, ${ }^{20}$ so introduction of tetraethylene glycol as linker may enhance the anti-TB activity.

Based on the above research results, a series of novel tetraethylene glycol tethered homonuclear and heteronuclear isatin dimers were designed and synthesized in this study. These isatin dimers were also evaluated for their in vitro anti-TB activity against MTB $\mathrm{H}_{37} \mathrm{Rv}$ and MDR-TB, as well as cytotoxicity in VERO cell line. The illustration of the design strategy is depicted in Figure 1.

\section{RESULTS AND DISCUSSION}

Detailed synthetic route for tetraethylene glycol tethered homonuclear isatin dimers 5a-d and heteronuclear dimers 10a-f were depicted in Scheme 1 and 2, respectively. Intermediate $\mathbf{2}$ was obtained by treatment of tetraethylene glycol $\mathbf{1}$ with tosyl chloride (3 eq) in presence of triethylamine. C-5 substituted isatin $\mathbf{3}$ was alkylated with intermediate 2 in presence of $\mathrm{K}_{2} \mathrm{CO}_{3}$ to provide the desired targets $\mathbf{4 a}, \mathbf{b}$, which were consequently condensation with semicarbazide or thiosemicarbazide hydrochloride in the presence of $\mathrm{NaHCO}_{3}$ provided targets 5a-d. ${ }^{21,22}$

5-Methylisatin 6 was reacted with the requested amine hydrochloride with potassium carbonate as base yielded the intermediates 7 . Intermediate 9 was obtained by treatment of 5-fluoroisatin 8 with intermediate $\mathbf{2}$, and intermediate $\mathbf{9}$ was consequently utilized for the synthesis of desired tetraethylene glycol tethered heteronuclear isatin dimers $\mathbf{1 0 a}, \mathbf{b}$ with potassium carbonate as base. Finally, condensations of targets $\mathbf{1 0 a}, \mathbf{b}$ with hydroxylamine or methoxyamine or ethoxyamine hydrochloride in the presence of sodium bicarbonate provided targets 10c-f $(43 \sim 68 \%))^{21}$

All tetraethylene glycol tethered homonuclear isatin dimers 5a-d and heteronuclear dimers 10a-f were screened for their in vitro anti-mycobacterial activities against MTB H37Rv and MDR-TB strains as well as cytotoxicity in VERO cell line, and the results were listed in Table 1. The MDR-TB strain was resistant to INH, RIF and EMB. The minimum inhibitory concentration (MIC) is defined as the lowest concentration that inhibits the visible bacterial growth.

The preliminary results indicated that all isatin dimers displayed considerable anti-mycobacterial activities against the tested strains with MIC in a range of 32 to $512 \mu \mathrm{g} / \mathrm{mL}$, and the potential activity may be partly attributed to their hydrogen bonding capacity. The structure-activity relationship (SAR) revealed that the anti-mycobacterial activity was greatly influenced by the substituents at C-3 and C-5 positions of isatin moiety: for $\mathrm{C}-3$ position, the relative contribution order of the substituents to the activity was as follows: $-\mathrm{NOMe}>-\mathrm{NOEt}>-\mathrm{O}>-$ $\mathrm{NOH}$ and $-\mathrm{NNHCSNH}_{2}>-\mathrm{NNHCONH}_{2}$; introduction of electron-withdrawing group -F at $\mathrm{C}-5$ position of isatin motif favored the activity. In particular, the isatin dimer 10f (MIC: 32 and $32 \mu \mathrm{g} / \mathrm{mL}$ ) was found to be most active against MTB $\mathrm{H}_{37} \mathrm{Rv}$ and MDR-TB strains, which was comparable to or better than the first-line anti-TB agents RIF (MIC: $32 \mu \mathrm{g} / \mathrm{mL}$ ) and INH (MIC: $>128 \mu \mathrm{g} / \mathrm{mL}$ ) against MDR-TB, could act as a lead for further optimization.

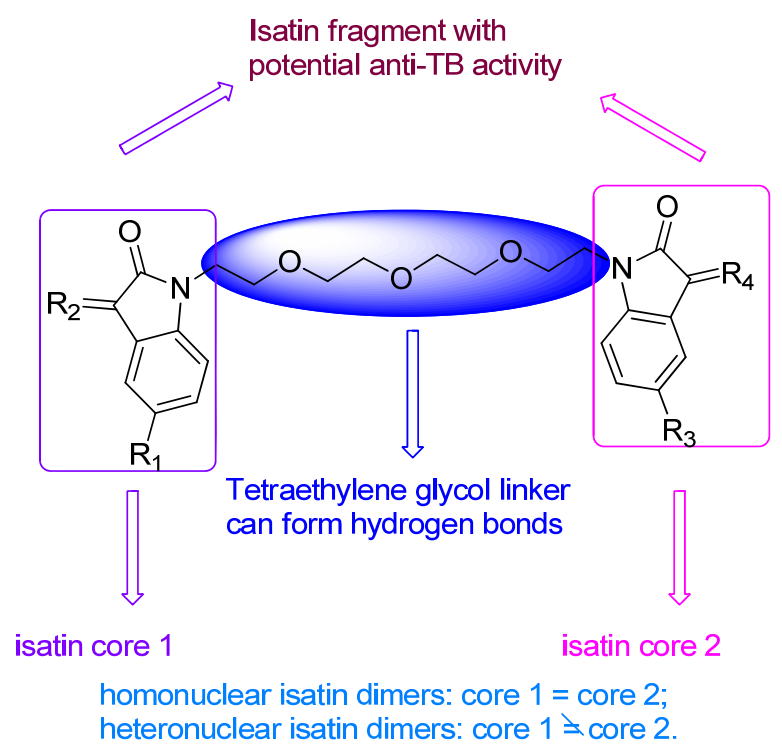

Fig. 1 - Design strategy of tetraethylene glycol tethered homonuclear and heteronuclear isatin dimers. 


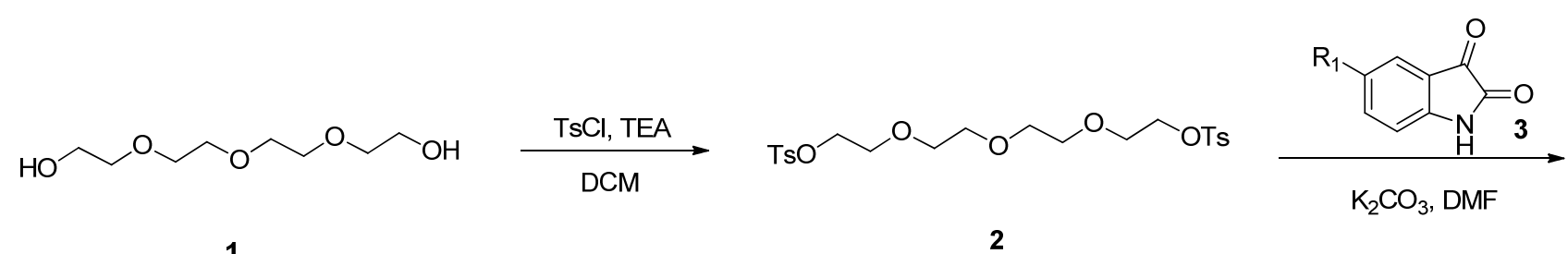

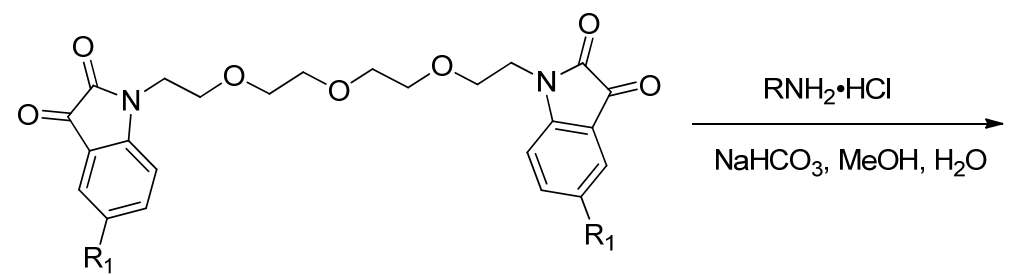

$4 a, b$

4a: $R_{1}=H ; 4 b: R_{1}=F$.<smiles>[R]c1ccc2c(c1)c([R2])c([R])n2CCOCCOCCOCCN1CC(=O)C([R2])C1=O</smiles>

$5 a-d$

5a: $\mathrm{R}_{1}=\mathrm{H}, \mathrm{R}_{2}=\mathrm{NNHCONH}_{2}$;

5b: $\mathrm{R}_{1}=\mathrm{H}, \mathrm{R}_{2}=\mathrm{NNHCSNH}_{2}$;

5c: $R_{1}=F, R_{2}=N_{N H C O N H}$;

5d: $R_{1}=F, R_{2}=N_{N H C S N H}$.

Scheme 1 - Synthesis of tetraethylene glycol tethered homonuclear isatin dimers 5a-d.<smiles>Cc1ccc2c(c1)C(=O)C(=O)N2</smiles>

6

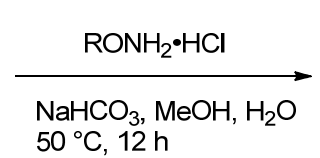

$50^{\circ} \mathrm{C}, 12 \mathrm{~h}$<smiles>[R]C1C(=O)Nc2ccc(C)cc21</smiles>

7

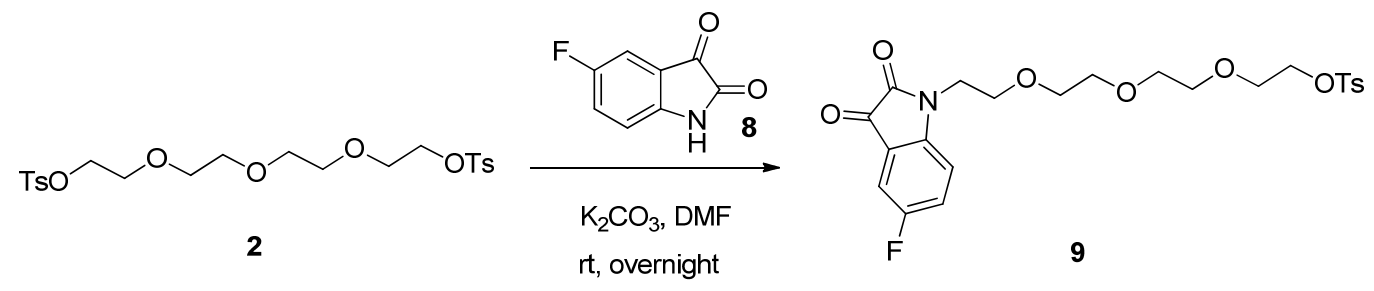<smiles>[R]C(=O)O[Na]</smiles>

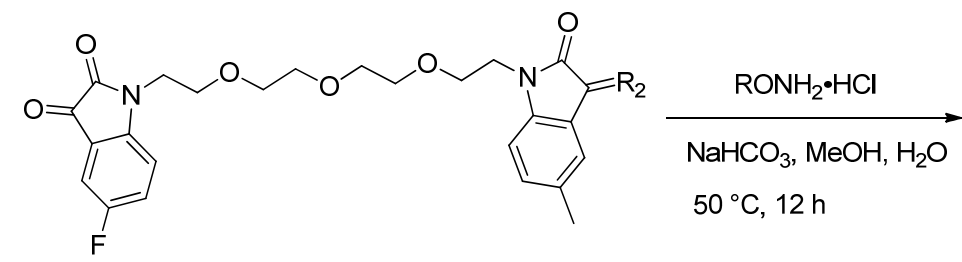

10a,b<smiles></smiles>

10c-f

10a: $R_{2}=$ NOMe;10b: $R_{2}=$ NOEt.

10c: $R_{1}=N O H, R_{2}=N O E t ;$

10d: $R_{1}=$ NOMe, $R_{2}=$ NOEt;

10e: $R_{1}=$ NOEt, $R_{2}=$ NOEt;

10f: $R_{1}=$ NOMe, $R_{2}=$ NOMe.

Scheme 2 - Synthesis of heteronuclear isatin dimers 10a-f. 
Table 1

Structures, anti-mycobacterial activities and cytotoxicity of homonuclear isatin dimers 5a-d and heteronuclear dimers 10a-f<smiles>[R]C1=C(C)N(CCOCCOCCOCCn2c([R])c([R])c3cc([R3])ccc32)C1=O</smiles>

\begin{tabular}{|c|c|c|c|c|c|c|c|}
\hline \multirow{2}{*}{ Compd. } & \multirow{2}{*}{$\mathrm{R}_{1}$} & \multirow{2}{*}{$\mathrm{R}_{2}$} & \multirow{2}{*}{$\mathrm{R}_{3}$} & \multirow{2}{*}{$\mathrm{R}_{4}$} & \multicolumn{2}{|c|}{$\operatorname{MIC}(\mu \mathrm{g} / \mathrm{mL})$} & \multirow{2}{*}{${ }^{\mathrm{b}} \mathrm{CC}_{50}(\mu \mathrm{g} / \mathrm{mL})$} \\
\hline & & & & & ${ }^{\mathrm{a}} \mathrm{MTB}$ & MDR-TB & \\
\hline $5 \mathbf{a}$ & $\mathrm{H}$ & $\mathrm{NNHCONH}_{2}$ & $\mathrm{H}$ & $\mathrm{NNHCONH}_{2}$ & 128 & 256 & 62.5 \\
\hline $5 b$ & $\mathrm{H}$ & $\mathrm{NNHCSNH}_{2}$ & $\mathrm{H}$ & $\mathrm{NNHCSNH}_{2}$ & 128 & 128 & 31.2 \\
\hline $5 c$ & $\mathrm{~F}$ & $\mathrm{NNHCONH}_{2}$ & $\mathrm{~F}$ & $\mathrm{NNHCONH}_{2}$ & 64 & 128 & 31.2 \\
\hline $5 d$ & F & $\mathrm{NNHCSNH}_{2}$ & F & $\mathrm{NNHCSNH}_{2}$ & 64 & 32 & 15.6 \\
\hline $10 \mathrm{a}$ & $\mathrm{F}$ & $\mathrm{O}$ & $\mathrm{Me}$ & NOMe & 128 & 128 & 62.5 \\
\hline $10 \mathrm{~b}$ & $\mathrm{~F}$ & $\mathrm{O}$ & $\mathrm{Me}$ & NOEt & 128 & 256 & 62.5 \\
\hline $10 \mathrm{c}$ & $\mathrm{F}$ & $\mathrm{NOH}$ & $\mathrm{Me}$ & NOEt & 256 & 512 & 125 \\
\hline $10 d$ & $\mathrm{~F}$ & $\mathrm{NOMe}$ & $\mathrm{Me}$ & NOEt & 64 & 64 & 31.2 \\
\hline $10 \mathrm{e}$ & $\mathrm{F}$ & NOEt & $\mathrm{Me}$ & NOEt & 128 & 64 & 125 \\
\hline $10 \mathrm{f}$ & $\mathrm{F}$ & NOMe & $\mathrm{Me}$ & $\mathrm{NOMe}$ & 32 & 32 & 250 \\
\hline INH & & & & & 0.05 & $>128$ & 125 \\
\hline RIF & & & & & 0.39 & 32 & 500 \\
\hline
\end{tabular}

${ }^{a}$ MTB: MTB H37Rv

${ }^{\mathrm{b}} \mathrm{CC}_{50}$ : The $50 \%$ cytotoxic concentration in a mammalian VERO cell line.

Interestingly, the resistance index (RI) for a significant part of tetraethylene glycol tethered isatin dimers was around 1, suggesting they may bear a novel action mechanism.

The isatin dimers were subsequently examined for toxicity (the $50 \%$ cytotoxic concentration $/ \mathrm{CC}_{50}$, causing visible morphological changes in $50 \%$ of the cells with respect to cell control, determined with the 3-(4,5-dimethylthiazol-2-yl)-2,5-diphenyltetrazolium bromide cell viability assay) in a mammalian VERO cell line. ${ }^{21}$ After $72 \mathrm{~h}$ of exposure, viability was assessed on the basis of cellular conversion of MTT (3-(4,5-dimethylthiazol-2-yl)-2,5-diphenyltetrazolium bromide) into a formazan product and the results were reported in Table 1. The results showed that all isatin dimers showed acceptable cytotoxicity against VERO cell line with $\mathrm{CC}_{50}$ of $15.6-250 \mu \mathrm{g} / \mathrm{mL}$. The most active isatin dimer 10f $\left(\mathrm{CC}_{50}: 250 \mu \mathrm{g} / \mathrm{mL}\right)$ showed the lowest cytotoxicity, which was comparable to RIF (MIC: $500 \mu \mathrm{g} / \mathrm{mL}$ ) and INH (MIC: $125 \mu \mathrm{g} / \mathrm{mL}$ ), warrant further investigation.

\section{EXPERIMENTAL}

The general procedure

for preparing targets 5a-d and 10a-f

A mixture of tetraethylene glycol 1 (10 mmol), tosyl chloride $(30 \mathrm{mmol})$ and triethylamine $(50 \mathrm{mmol})$ in DCM (100 $\mathrm{mL}$ ) was stirred at room temperature overnight, and then concentrated under reduced pressure. The residue was purified by silica gel chromatography eluted with $\mathrm{PE}: \mathrm{EA}=1: 2$ to give the intermediate 2 . 
The mixture of intermediate 2 ( $1 \mathrm{mmol})$, potassium carbonate $(10 \mathrm{mmol})$ and isatins $3(0.5 \mathrm{mmol})$ in DMF $(10 \mathrm{~mL})$ was stirred at room temperature overnight. After filtration, the filtrate was concentrated under reduced pressure. The residue was purified by silica gel chromatography eluted with PE:EA=1:2 to give the key intermediates $\mathbf{4 a , b}$. To a mixture of intermediates $\mathbf{4 a , b}(1 \mathrm{mmol})$ in water $(10 \mathrm{~mL})$ and THF $(50 \mathrm{~mL})$, the requested amine hydrochloride $(5 \mathrm{mmol})$ was added. The mixture was stirred at $50{ }^{\circ} \mathrm{C}$ for $12 \mathrm{~h}$. After cooling to room temperature, the mixture was extracted with EA $\left(100 \mathrm{~mL}^{*} 2\right)$. The combined organic layers were washed with $\mathrm{H}_{2} \mathrm{O}\left(100 \mathrm{~mL}^{*}\right)$ and brine $(100 \mathrm{~mL})$ in sequence, and dried over $\mathrm{Na}_{2} \mathrm{SO}_{4}$. After filtration, the filtrate was concentrated under reduced pressure to give a residue which was purified by silica gel chromatography eluted with $\mathrm{PE}: \mathrm{EA}=1: 2$ to give the homonuclear isatin dimers 5a-d.

A mixture of 5-methylisatin $6(1 \mathrm{mmol})$, potassium carbonate $(5 \mathrm{mmol})$ and methoxyamine or ethoxyamine hydrochloride $(1.2 \mathrm{mmol})$ in a mixture of water $(5 \mathrm{~mL})$ and THF $(30 \mathrm{~mL})$ was stirred at $50{ }^{\circ} \mathrm{C}$ for $12 \mathrm{~h}$. After cooling to room temperature, the mixture was extracted with EA (30 $\mathrm{mL} * 2$ ). The combined organic layers were washed with $\mathrm{H}_{2} \mathrm{O}$ $(20 \mathrm{~mL} * 2)$ and brine $(20 \mathrm{~mL})$ in sequence, dried over $\mathrm{Na}_{2} \mathrm{SO}_{4}$, filtrated, and concentrated under reduced pressure. The crude intermediates 7 were obtained as a yellow solid which were used directly without further purification.

The mixture of intermediate $2(2 \mathrm{mmol})$, potassium carbonate $(10 \mathrm{mmol})$ and 5 -fuloroisatin $8(1 \mathrm{mmol})$ in DMF $(10 \mathrm{~mL})$ was stirred at room temperature overnight. After filtration, the filtrate was concentrated under reduced pressure. The residue was purified by silica gel chromatography eluted with PE:EA=1:2 to give the intermediates 9. A mixture of intermediate 9 (1 mmol), intermediate $7(1 \mathrm{mmol})$ and potassium carbonate $(10 \mathrm{mmol})$ in DMF $(10 \mathrm{~mL})$ was stirred at room temperature for 2 days. After filtration, the filtrate was concentrated under reduced pressure. The residue was purified by silica gel chromatography eluted with $P E: E A=1: 2$ to give the desired heteronuclear isatin dimers $\mathbf{1 0 a}, \mathbf{b}$.

To a mixture of heteronuclear isatin dimers 10a,b $(1 \mathrm{mmol})$ in water $(10 \mathrm{~mL})$ and THF $(50 \mathrm{~mL})$, methoxyamine or ethoxyamine hydrochloride $(1.4 \mathrm{mmol})$ was added. The mixture was stirred at $50{ }^{\circ} \mathrm{C}$ for $12 \mathrm{~h}$. After cooling to room temperature, the mixture was extracted with EA $\left(100 \mathrm{~mL}^{*} 2\right)$. The combined organic layers were washed with $\mathrm{H}_{2} \mathrm{O}$ $\left(100 \mathrm{~mL}^{*} 2\right)$ and brine $(100 \mathrm{~mL})$ in sequence, and dried over $\mathrm{Na}_{2} \mathrm{SO}_{4}$. After filtration, the filtrate was concentrated under reduced pressure to give a residue which was purified by silica gel chromatography eluted with $\mathrm{PE}: \mathrm{EA}=1: 2$ to give the desired products 10c-f.

1.1. $1,1^{\prime}-((($ oxybis (ethane-2,1-diyl))bis(oxy))bis(ethane2,1-diyl))bis(3-(ethoxyimino)indolin-2-one) (5a)

Yellow solid, yield: $52 \%$. ${ }^{1} \mathrm{H}$ NMR (400 MHz, DMSO- $d_{6}$ ) $1.38\left(6 \mathrm{H}, \mathrm{t}, 2 \times \mathrm{NOCH}_{2} \mathrm{CH}_{3}\right), 3.38\left(4 \mathrm{H}, \mathrm{t},-\mathrm{CH}_{2} \mathrm{CH}_{2}-\right), 3.44$ $\left(4 \mathrm{H}, \mathrm{t},-\mathrm{CH}_{2} \mathrm{CH}_{2}-\right), 3.62\left(4 \mathrm{H}, \mathrm{t},-\mathrm{CH}_{2} \mathrm{CH}_{2}-\right), 3.86(4 \mathrm{H}, \mathrm{t},-$ $\left.\mathrm{CH}_{2} \mathrm{CH}_{2}-\right), 4.48\left(4 \mathrm{H}, \mathrm{q}, 2 \times \mathrm{NOCH}_{2} \mathrm{CH}_{3}\right), 7.06(2 \mathrm{H}, \mathrm{t}, \mathrm{Ar}-\mathrm{H})$, $7.15(2 \mathrm{H}, \mathrm{d}, \mathrm{Ar}-\mathrm{H}), 7.44(2 \mathrm{H}, \mathrm{t}, \mathrm{Ar}-\mathrm{H}), 7.88(2 \mathrm{H}, \mathrm{d}, \mathrm{Ar}-\mathrm{H})$. ESI-MS m/z: $539[\mathrm{M}+\mathrm{H}]^{+}, 561[\mathrm{M}+\mathrm{Na}]^{+}$

1.2. $2,2^{\prime}-\left(1,1^{\prime}-(((\right.$ oxybis (ethane-2,1-diyl) )bis(oxy)) bis(ethane-2,1-diyl))bis(2-oxoindoline-1-yl-3-ylidene))bis(hydrazinecarboxamide) $(\mathbf{5 b})$

Yellow solid, yield: $17 \% .{ }^{1} \mathrm{H}$ NMR $\left(400 \mathrm{MHz}, \mathrm{DMSO}-d_{6}\right)$ $3.38\left(4 \mathrm{H}, \mathrm{t},-\mathrm{CH}_{2} \mathrm{CH}_{2}-\right), 3.45\left(4 \mathrm{H}, \mathrm{t},-\mathrm{CH}_{2} \mathrm{CH}_{2}-\right), 3.66(4 \mathrm{H}, \mathrm{t},-$ $\left.\mathrm{CH}_{2} \mathrm{CH}_{2-}\right), 3.92\left(4 \mathrm{H}, \mathrm{t},-\mathrm{CH}_{2} \mathrm{CH}_{2}-\right), 7.12-7.20(8 \mathrm{H}, \mathrm{m}$, $2 \times \mathrm{NNHCONH}_{2}$ and $\left.\mathrm{Ar}-\mathrm{H}\right), 7.38(2 \mathrm{H}, \mathrm{t}, \mathrm{Ar}-\mathrm{H}), 7.65(2 \mathrm{H}, \mathrm{d}$, Ar-H), $11.70\left(2 \mathrm{H}, \mathrm{s}, 2 \times \mathrm{NNHCONH}_{2}\right)$. ESI-MS m/z: 589 $[\mathrm{M}+\mathrm{Na}]^{+}$.
1.3. $2,2^{\prime}-\left(1,1^{\prime}-((\right.$ oxybis (ethane- $2,1-$ diyl))bis(oxy))bis(ethane-2,1-diyl))bis(5-fluoro-2-oxoindoline1-yl-3-ylidene))bis(hydrazinecarboxamide) (5c) Yellow solid, yield: $27 \%$. ${ }^{1} \mathrm{H}$ NMR $\left(400 \mathrm{MHz}, \mathrm{DMSO}-d_{6}\right) \delta$ $3.39\left(4 \mathrm{H}, \mathrm{t},-\mathrm{CH}_{2} \mathrm{CH}_{2}-\right), 3.47\left(4 \mathrm{H}, \mathrm{t},-\mathrm{CH}_{2} \mathrm{CH}_{2}-\right), 3.65(4 \mathrm{H}, \mathrm{t},-$ $\left.\mathrm{CH}_{2} \mathrm{CH}_{2-}\right), 3.92\left(4 \mathrm{H}, \mathrm{t},-\mathrm{CH}_{2} \mathrm{CH}_{2}-\right), 7.21-7.26(8 \mathrm{H}, \mathrm{m}, \mathrm{Ar}-\mathrm{H}$ and $\left.2 \times \mathrm{NNHCONH}_{2}\right), 7.50(2 \mathrm{H}, \mathrm{d}, \mathrm{Ar}-\mathrm{H}), 11.62(2 \mathrm{H}, \mathrm{s}$, $\left.2 \times \mathrm{NNHCONH}_{2}\right) . \mathrm{ESI}-\mathrm{MS} \mathrm{m} / \mathrm{z}: 625[\mathrm{M}+\mathrm{Na}]^{+}$.

1.4. $2,2^{\prime}-\left(1,1^{\prime}-(((\right.$ oxybis $($ ethane- $2,1-$

diyl))bis(oxy))bis(ethane-2,1-diyl))bis(5-fluoro-2-oxoindoline1-yl-3-ylidene))bis(hydrazinecarbothioamide) (5d)

Yellow solid, yield: $19 \%$. ${ }^{1} \mathrm{H}$ NMR $\left(400 \mathrm{MHz}\right.$, DMSO- $\left.d_{6}\right) \delta$ $3.40\left(4 \mathrm{H}, \mathrm{t},-\mathrm{CH}_{2} \mathrm{CH}_{2}-\right), 3.48\left(4 \mathrm{H}, \mathrm{t},-\mathrm{CH}_{2} \mathrm{CH}_{2}-\right), 3.67(4 \mathrm{H}, \mathrm{t},-$ $\left.\mathrm{CH}_{2} \mathrm{CH}_{2}-\right), 3.92\left(4 \mathrm{H}, \mathrm{t},-\mathrm{CH}_{2} \mathrm{CH}_{2}-\right), 7.24(4 \mathrm{H}, \mathrm{s}, \mathrm{Ar}-\mathrm{H}), 7.56$ $(2 \mathrm{H}, \mathrm{d}, \mathrm{Ar}-\mathrm{H}), 8.82,9.18\left(4 \mathrm{H}, \mathrm{s}, 2 \times \mathrm{NNHCSNH}_{2}\right), 12.32(2 \mathrm{H}$, s, $\left.2 \times \mathrm{NNHCSNH}_{2}\right)$. ESI-MS m/z: $635[\mathrm{M}+\mathrm{H}]^{+}$.

1.5. 5-fluoro-1-(2-(2-(2-(2-(3-(methoxyimino)-5-methyl-2oxoindolin-1-yl)ethoxy)ethoxy)ethoxy)ethyl)indoline-2,3-dione (10a)

Yellow solid, yield: $83 \% .{ }^{1} \mathrm{H}$ NMR $\left(400 \mathrm{MHz}, \mathrm{DMSO}-d_{6}\right) \delta$ $2.29\left(3 \mathrm{H}, \mathrm{s},-\mathrm{CH}_{3}\right), 3.42\left(4 \mathrm{H}, \mathrm{t},-\mathrm{CH}_{2} \mathrm{CH}_{2}-\right), 3.48(4 \mathrm{H}, \mathrm{t},-$ $\left.\mathrm{CH}_{2} \mathrm{CH}_{2}-\right), 3.67\left(4 \mathrm{H}, \mathrm{t},-\mathrm{CH}_{2} \mathrm{CH}_{2}-\right), 3.84\left(4 \mathrm{H}, \mathrm{t},-\mathrm{CH}_{2} \mathrm{CH}_{2}-\right)$, $4.21(3 \mathrm{H}, \mathrm{s}, \mathrm{NOMe}), 7.06(1 \mathrm{H}, \mathrm{d}, \mathrm{Ar}-\mathrm{H}), 7.24-7.27(2 \mathrm{H}, \mathrm{m}$, Ar-H), $7.44(1 \mathrm{H}, \mathrm{d}, \mathrm{Ar}-\mathrm{H}), 7.50(1 \mathrm{H}, \mathrm{t}, \mathrm{Ar}-\mathrm{H}), 7.70(1 \mathrm{H}, \mathrm{s}, \mathrm{Ar}-$ H). ESI-MS m/z: $536[\mathrm{M}+\mathrm{Na}]^{+}$.

1.6. $1-(2-(2-(2-(2-(3-($ ethoxyimino $)-5-m e t h y l-2-o x o i n d o l i n-1-$ yl)ethoxy)ethoxy)ethoxy)ethyl)-5-fluoroindoline-2,3-dione (10b) Yellow solid, yield: $72 \%$. ${ }^{1} \mathrm{H}$ NMR $\left(400 \mathrm{MHz}, \mathrm{DMSO}-d_{6}\right) \delta$ $1.40\left(3 \mathrm{H}, \mathrm{t}, \mathrm{NOCH}_{2} \mathrm{CH}_{3}\right), 2.29\left(3 \mathrm{H}, \mathrm{s},-\mathrm{CH}_{3}\right), 3.41(4 \mathrm{H}, \mathrm{t},-$ $\left.\mathrm{CH}_{2} \mathrm{CH}_{2}-\right), 3.48\left(4 \mathrm{H}, \mathrm{t},-\mathrm{CH}_{2} \mathrm{CH}_{2}-\right), 3.62\left(4 \mathrm{H}, \mathrm{t},-\mathrm{CH}_{2} \mathrm{CH}_{2}-\right)$, $3.84\left(4 \mathrm{H}, \mathrm{t},-\mathrm{CH}_{2} \mathrm{CH}_{2}-\right), 4.47\left(4 \mathrm{H}, \mathrm{q}, 2 \times \mathrm{NOCH}_{2} \mathrm{CH}_{3}\right), 7.05$ $(1 \mathrm{H}, \mathrm{t}, \mathrm{Ar}-\mathrm{H}), 7.17-7.31(3 \mathrm{H}, \mathrm{m}, \mathrm{Ar}-\mathrm{H}), 7.43-7.50(1 \mathrm{H}, \mathrm{m}, \mathrm{Ar}-$ $\mathrm{H}), 7.65-7.71(1 \mathrm{H}, \mathrm{m}, \mathrm{Ar}-\mathrm{H})$. ESI-MS m/z: $550[\mathrm{M}+\mathrm{Na}]^{+}$.

1.7. 1-(2-(2-(2-(2-(2-(ethoxyimino)-5-methyl-3-oxoindolin1-yl)ethoxy)ethoxy)ethoxy)ethyl)-5-fluoro-3(hydroxyimino)indolin-2-one (10c)

Yellow solid, yield: $51 \%$. ${ }^{1} \mathrm{H}$ NMR (400 MHz, DMSO- $\left.d_{6}\right)$ $1.40\left(3 \mathrm{H}, \mathrm{t}, \mathrm{NOCH}_{2} \mathrm{CH}_{3}\right), 2.31\left(3 \mathrm{H}, \mathrm{s},-\mathrm{CH}_{3}\right), 3.42(4 \mathrm{H}, \mathrm{t},-$ $\left.\mathrm{CH}_{2} \mathrm{CH}_{2}-\right), 3.49\left(4 \mathrm{H}, \mathrm{t},-\mathrm{CH}_{2} \mathrm{CH}_{2^{-}}\right), 3.64\left(4 \mathrm{H}, \mathrm{t},-\mathrm{CH}_{2} \mathrm{CH}_{2}-\right)$, $3.87\left(4 \mathrm{H}, \mathrm{t},-\mathrm{CH}_{2} \mathrm{CH}_{2}-\right), 4.49\left(4 \mathrm{H}, \mathrm{q}, 2 \times \mathrm{NOCH}_{2} \mathrm{CH}_{3}\right), 7.07$ $(1 \mathrm{H}, \mathrm{d}, \mathrm{Ar}-\mathrm{H}), 7.20(1 \mathrm{H}, \mathrm{d}, \mathrm{Ar}-\mathrm{H}), 7.27-7.30(2 \mathrm{H}, \mathrm{m}, \mathrm{Ar}-\mathrm{H})$, 7.73-7.78 (2H, m, Ar-H), $13.73(1 \mathrm{H}, \mathrm{s}, \mathrm{NOH})$. ESI-MS m/z: $565[\mathrm{M}+\mathrm{Na}]^{+}$

1.8. 1-(2-(2-(2-(2-(2-(ethoxyimino)-5-methyl-3-oxoindolin1-yl)ethoxy)ethoxy)ethoxy)ethyl)-5-fluoro-3-

(methoxyimino)indolin-2-one (10d)

Yellow solid, yield: $61 \%$. ${ }^{1} \mathrm{H}$ NMR $\left(400 \mathrm{MHz}, \mathrm{DMSO}-d_{6}\right)$ $1.40\left(3 \mathrm{H}, \mathrm{t}, \mathrm{NOCH}_{2} \mathrm{CH}_{3}\right), 2.28\left(3 \mathrm{H}, \mathrm{s},-\mathrm{CH}_{3}\right), 3.40(4 \mathrm{H}, \mathrm{t},-$ $\left.\mathrm{CH}_{2} \mathrm{CH}_{2}-\right), 3.46\left(4 \mathrm{H}, \mathrm{t},-\mathrm{CH}_{2} \mathrm{CH}_{2}-\right), 3.61\left(4 \mathrm{H}, \mathrm{t},-\mathrm{CH}_{2} \mathrm{CH}_{2}-\right)$, $3.83\left(4 \mathrm{H}, \mathrm{t},-\mathrm{CH}_{2} \mathrm{CH}_{2}-\right), 4.24-4.50(5 \mathrm{H}, \mathrm{m}, \mathrm{NOMe}$ and $\left.\mathrm{NOCH}_{2} \mathrm{CH}_{3}\right), 7.03-7.30(4 \mathrm{H}, \mathrm{m}, \mathrm{Ar}-\mathrm{H}), 7.64(1 \mathrm{H}, \mathrm{d}, \mathrm{Ar}-\mathrm{H})$, $7.69(1 \mathrm{H}, \mathrm{d}, \mathrm{Ar}-\mathrm{H}) . \mathrm{ESI}-\mathrm{MS} \mathrm{m} / \mathrm{z}: 579[\mathrm{M}+\mathrm{Na}]^{+}$.

1.9. 3-(ethoxyimino)-1-(2-(2-(2-(2-(2-(ethoxyimino)-5methyl-3-oxoindolin-1-yl)ethoxy)ethoxy)ethoxy)ethyl)-5fluoroindolin-2-one (10e)

Yellow solid, yield: $45 \%$. ${ }^{1} \mathrm{H}$ NMR $\left(400 \mathrm{MHz}\right.$, DMSO- $\left.d_{6}\right)$ $1.40\left(6 \mathrm{H}, \mathrm{t}, 2 \times \mathrm{NOCH}_{2} \mathrm{CH}_{3}\right), 2.29\left(3 \mathrm{H}, \mathrm{s},-\mathrm{CH}_{3}\right), 3.40(4 \mathrm{H}, \mathrm{t},-$ $\left.\mathrm{CH}_{2} \mathrm{CH}_{2}-\right), 3.46\left(4 \mathrm{H}, \mathrm{t},-\mathrm{CH}_{2} \mathrm{CH}_{2}-\right), 3.62\left(4 \mathrm{H}, \mathrm{t},-\mathrm{CH}_{2} \mathrm{CH}_{2}-\right)$, $3.85\left(4 \mathrm{H}, \mathrm{t},-\mathrm{CH}_{2} \mathrm{CH}_{2}-\right), 4.50\left(4 \mathrm{H}, \mathrm{q}, 2 \times \mathrm{NOCH}_{2} \mathrm{CH}_{3}\right), 7.05$ $(1 \mathrm{H}, \mathrm{d}, \mathrm{Ar}-\mathrm{H}), 7.19(1 \mathrm{H}, \mathrm{d}, \mathrm{Ar}-\mathrm{H}), 7.24(1 \mathrm{H}, \mathrm{d}, \mathrm{Ar}-\mathrm{H}), 7.31$ $(1 \mathrm{H}, \mathrm{d}, \mathrm{Ar}-\mathrm{H}), 7.64(1 \mathrm{H}, \mathrm{d}, \mathrm{Ar}-\mathrm{H}), 7.71(1 \mathrm{H}, \mathrm{d}, \mathrm{Ar}-\mathrm{H})$. ESIMS m/z: $593[\mathrm{M}+\mathrm{Na}]^{+}$.

1.10. 5-fluoro-3-(methoxyimino)-1-(2-(2-(2-(2-(2-(methoxyimino)-5-methyl-3-oxoindolin-1yl)ethoxy)ethoxy)ethoxy)ethyl)indolin-2-one (10f) Yellow solid, yield: 74\%. ${ }^{1} \mathrm{H}$ NMR (400 MHz, DMSO- $\left.d_{6}\right)$ $2.29\left(3 \mathrm{H}, \mathrm{s},-\mathrm{CH}_{3}\right), 3.40\left(4 \mathrm{H}, \mathrm{t},-\mathrm{CH}_{2} \mathrm{CH}_{2}-\right), 3.47(4 \mathrm{H}, \mathrm{t},-$ 
$\left.\mathrm{CH}_{2} \mathrm{CH}_{2}-\right), 3.62\left(4 \mathrm{H}, \mathrm{t},-\mathrm{CH}_{2} \mathrm{CH}_{2}-\right), 3.85\left(4 \mathrm{H}, \mathrm{t},-\mathrm{CH}_{2} \mathrm{CH}_{2}-\right)$, $4.28(6 \mathrm{H}, \mathrm{s}, 2 \times \mathrm{NOMe}), 7.06(1 \mathrm{H}, \mathrm{s}, \mathrm{Ar}-\mathrm{H}), 7.19-7.33(3 \mathrm{H}, \mathrm{m}$, Ar-H), 7.66-7.69 (2H, m, Ar-H). ESI-MS m/z: $565[\mathrm{M}+\mathrm{Na}]^{+}$.

\section{MIC determination}

The homonuclear isatin dimers 5a-d and heteronuclear dimers 10a-f along with the references RIF and INH were evaluated for their in vitro activities against MTB H37Rv and MDR-TB via rapid direct susceptibility test technique. ${ }^{21,22}$ MTB H37Rv and MDR-TB strains were obtained from Chinese Center For Disease Control And Prevention. The wells of a sterile 48-well plate were filled with $100 \mathrm{~mL}$ twofold diluted tested compounds and $100 \mathrm{~mL}$ MTB H37Rv or MDR-TB suspension containing $4 \times 10^{-3} \mathrm{mg}$ cells. Pure medium replaced the diluted compounds in two wells as the positive control of growth, and deionized water instead of the culture in other two wells as the negative control of growth in the plates. The plates were covered and sealed, then incubated at $37^{\circ} \mathrm{C}$ in a wet box. The positive and negative control wells should show obvious difference after 3 days. The MIC was determined by observing the quantity and state of the cells in each test well by a continuous visual high magnification system, and re-determined 7 days later. The MIC is defined as the concentration of the compound required to give complete inhibition of bacterial growth.

\section{Cytotoxicity}

The synthesized homonuclear isatin dimers 5a-d and heteronuclear dimers 10a-f along with the references RIF and INH were further examined for toxicity $\left(\mathrm{CC}_{50}\right)$ determined with the 3-(4,5-dimethylthiazol-2-yl)-2,5-diphenyltetrazolium bromide cell viability assay in a mammalian VERO cells (obtained from Chinese Center For Disease Control And Prevention). ${ }^{21}$ The VERO cells were maintained in culture medium (Minimum Essential Medium with Earle's salt, supplemented with 10\% fetal bovine serum) at $37{ }^{\circ} \mathrm{C}$ under $5 \% \mathrm{CO}_{2}$. Cells were seeded in 96-well plates at the plating density of $1 \times 10^{4}$ cells per well and allowed to recover for $24 \mathrm{~h}$. Culture medium was replaced by assay medium containing the compound to be tested or drugfree. After $72 \mathrm{~h}$ of exposure, cells were harvested and cell viability was assessed by $\mathrm{MTT}$ assay. The $\mathrm{CC}_{50}$ values were calculated by Bliss analyses.

\section{CONCLUSIONS}

In summary, a series novel tetraethylene glycol tethered homonuclear and heteronuclear isatin dimers were designed, synthesized and examined for their in vitro anti-mycobacterial activities against MTB H37Rv and MDR-TB as well as cytotoxicity in this paper. All the synthesized homonuclear and heteronuclear isatin dimers exhibited promising activities against the tested strains and excellent cytotoxicity profile. The most active isatin dimer 10f also showed the lowest cytotoxicity, and it could act as a starting point for further optimization.
Acknowledgements: This work was supported by the National Natural Science Foundation of China (Grant No. 51801068, 51701189), the Science and Technology Planning Project in Henan Province (Grant No. 132300410476) and Key Scientific Research projects of Colleges and Universities in Henan Province (Grant No. 19A430019).

\section{REFERENCES}

1. World Health Organization, Global Tuberculosis Report 2017.

2. S. Zhang, Z. Xu, C. Gao, Q. C. Ren, L. Chang, Z. S. Lv and L. S. Feng, Eur. J. Med. Chem., 2017, 138, 501.

3. Z. Xu, C. Gao, Q. C. Ren, X. F. Song, L. S. Feng and Z. S. Lv, Eur. J. Med. Chem., 2017, 139, 429

4. Y. Q. Hu, S. Zhang, F. Zhao, C. Gao, L. S. Feng, Z. S. Lv, Z. Xu and X. Wu, Eur. J. Med. Chem., 2017, 133, 255

5. Y. Q. Hu, Z. Xu, S. Zhang, X. Wu, J. W. Ding, Z. S. Lv and L. S. Feng, Eur. J. Med. Chem., 2017, 136, 122

6. Y. Q. Hu, S. Zhang, Z. Xu, Z. S. Lv, M. L. Liu and L. S. Feng, Eur. J. Med. Chem., 2017, 141, 335

7. G. F. Zhang, S. Zhang, B. F. Pan, M. L. Liu and L. S. Feng, Eur. J. Med. Chem., 2018, 143, 710

8. M. T. Gabr, N. S. El-Gohary, E. R. Elbendary, M. M. ElKerdawy and N. Ni, Eur. J. Med. Chem., 2017, 128, 36

9. Y. Q. Hu, C. Gao, S. Zhang, L. Xu, Z. Xu, L. S. Feng, X. Wu and F. Zhao, Eur. J. Med. Chem., 2017, 139, 22

10. Z. Xu, S. Zhang, C. Gao, F. Zhao, Z. S. Lv and L. S. Feng, Chin. Chem. Lett., 2017, 28, 159

11. Z. Xu, X. F. Song, Y. Q. Hu, M. Qiang and Z. S. Lv, Eur. J. Med. Chem., 2017, 138, 66

12. Z. Xu, S. Zhang, X. F. Song, M. Qiang and Z. S. Lv, Bioorg. Med. Chem. Lett., 2017, 27, 3643

13. X. H. Jin, Y. Xu, X. H. Yang, X. L. Chen, M. H. Wu, J. G. Guan and L. S. Feng, Curr. Top. Med. Chem., 2017, 17, 3213.

14. Z. Xu, X. F. Song, M. Qiang and Z. S. Lv, J. Heterocyclic Chem., 2017, 54, 3735 .

15. H. S. Ibrahim, S. M. Abou-Seri, N. S. M. Ismail, M. M. Elaasser, M. H. Aly and H. A. Abdel-Aziz, Eur. J. Med. Chem., 2016, 108, 415

16. A. Singh, T. Bains, H. J. Hahn, N. Liu, C. Tam, L. W. Cheng, K. M. Land and V. Kumar, MedChemComm, 2017, 8, 1982

17. G. F. Zhang, X. F. Liu, S. Zhang, B. F. Pan and M. L. Liu, Eur. J. Med. Chem., 2017, 146, 599

18. Y. Q. Hu, X. F. Song and J. Fan, J. Heterocyclic Chem., 2018, 55, 265

19. Y. Xu, J. G. Guan, Z. Xu and S. J. Zhao, Fitoterapia, 2018, 127, 383

20. G. C. Xu, Y. Jie, Q. C. Liu, Z. M. Huang, G. Y. Liang and B. X. Xu, J. Chin. Universities., 2016, 37, 1451.

21. L. S. Feng, M. L. Liu, S. Zhang, Y. Chai, B. Wang, Y. B. Zhang, K. Lv, Y. Guan, H. Y. Guo and C. L. Xiao, Eur. J. Med. Chem., 2011, 46, 341.

22. L. S. Feng, M. L. Liu, B. Wang, Y. Chai, X. Q. Hao, S. Meng and H. Y. Guo, Eur. J. Med. Chem., 2010, 45, 3407. 
